\title{
ON THE TOPOLOGY OF CONFORMALLY COMPACT EINSTEIN 4-MANIFOLDS
}

\author{
Sun-Yung A. Chang ${ }^{1}$, Jie Qing ${ }^{2}$ and Paul Yang ${ }^{3}$
}

\begin{abstract}
In this paper we study the topology of conformally compact Einstein 4-manifolds. When the conformal infinity has positive Yamabe invariant and the renormalized volume is also positive we show that the conformally compact Einstein 4-manifold will have at most finite fundamental group. Under the further assumption that the renormalized volume is relatively large, we conclude that the conformally compact Einstein 4-manifold is diffeomorphic to $B^{4}$ and its conformal infinity is diffeomorphic to $S^{3}$.
\end{abstract}

\section{INTRODUCTION}

Conformally compact Einstein manifolds play an important part in the AdS/CFT correspondence, a promising new area under extensive development in string theory $[\mathrm{Mc}][\mathrm{GKP}][\mathrm{W}]$. Mathematically the study of conformally compact Einstein structures began with the work of Fefferman and Graham ([FG1]) in which they gave a procedure to find local conformal invariants and the associated conformally covariant operators. It has been a very challenging problem to give general existence theory. Recently M. Anderson ([A2]) has shown the existence of conformally compact Einstein metrics whose boundary is the 3-sphere with arbitrary conformal structure in the connected component of the round one, thus extending the perturbation existence result of Graham and Lee ([GL]). On the other hand, in recent works, using several conformally covariant differential equations and their ties to Chern-Gauss-Bonnet formula, two of the coauthors with Gursky studied

\footnotetext{
${ }^{1}$ Princeton University, Department of Mathematics, Princeton, NJ 08544-1000, supported by NSF Grant DMS-0070542.

${ }^{2}$ University of California, Department of Mathematics, Santa Cruz, CA 95064, supported in part by NSF Grant DMS-0103160.

${ }^{3}$ Princeton University, Department of Mathematics, Princeton, NJ 08544-1000, supported by NSF Grant DMS-0070526.
} 
the conformal geometry of closed 4-manifolds and obtained remarkable progress in understanding of the topology of 4-manifolds. In our view, conformally compact Einstein 4-manifolds provide a platform to study conformal geometry of closed 3-manifolds. In this note we will at least use it to translate the results in four dimension to 3-manifolds.

An oriented manifold $(X, g)$ with boundary $M$ is a conformally compact Einstein manifold if there is a complete Einstein metric $g$ in the interior of $X$, and a smooth defining function $s$ for the boundary $M=\partial X=\{s=0\}$ so that $\left(X, s^{2} g\right)$ is a compact Riemannian manifold with boundary. Since defining functions are not unique the data $(X, g)$ determines a conformal structure $(M,[\hat{g}])$ which is called the conformal infinity of $(X, g)$. It will be advantageous to find a defining function $s$ whose associated conformal metric enjoys optimal positivity property. To this end, one of us first observe ([Q]),

Theorem. Suppose that $\left(X^{n+1}, g\right)$ is a conformally compact Einstein manifold, and that $\hat{g}$ is a Yamabe metric for $(M,[\hat{g}])$. Then there exists a conformal compactification $\left(X, u^{-2} g\right)$, which has a totally geodesic boundary $M$ and whose scalar curvature $R\left[u^{-2} g\right] \geq \frac{n+1}{n-1} R[\hat{g}]$.

An important invariant of the conformally compact Einstein structure is the renormalized volume (see section one for the precise definition). The volume of a conformally compact Einstein manifold is infinite. But an appropriate normalization gives rise to the new invariants as suggested in the works of Maldacena [Mc], Witten [W], and Gubser, Klebanov and Polyakov [GKP]. The renormalization was carried out by Henningson and Skenderis in [HS] and later also by Graham in [Gr]. Anderson in [A1] observed that the renormalized volume for a conformally compact Einstein 4-manifold appears in the Chern-Gauss-Bonnet formula

$$
8 \pi^{2} \chi(X)=\frac{1}{4} \int_{X}|\mathcal{W}|^{2} d v_{g}+6 V
$$

where $\mathcal{W}$ is the Weyl curvature and the norm of the Weyl tensor is given by $|\mathcal{W}|^{2}=$ $\mathcal{W}_{i j k l} \mathcal{W}^{i j k l}$; i.e., the usual definition when $W$ is viewed as a section of $\otimes^{4} T^{*} M^{4}$ and $V$ is the renormalized volume of a conformally compact Einstein 4-manifold $\left(X^{4}, g\right)$. It follows that the renormalized volume is an invariant of the underlying conformal structure of the conformally compact Einstein manifold. On the other hand, we recall that for a closed 4-manifold $(Y, g)$, we have the Chern-Gauss-Bonnet formula

$$
8 \pi^{2} \chi(Y)=\int_{Y}\left(\frac{1}{4}|\mathcal{W}|^{2}+\sigma_{2}\left(A_{g}\right)\right) d v_{g}
$$

where $A_{g}=R c-\frac{R}{6} g$ is the Weyl Schouten tensor and $\sigma_{2}\left(A_{g}\right)$ is the second symmetric function of the eigenvalues of $A_{g}$. Thus the renormalized volume is a combination 
of a boundary invariant as well as the Ricci part of the Chern-Gauss-Bonnet integral. According to the above cited Theorem, the given compactification $X$ has a totally geodesic boundary. It is then natural to consider the doubling manifold $Y$. In this setting, we will show that the renormalized volume plays an important role in restricting the topology and geometry of the conformally compact Einstein manifolds. In particular, we have

Theorem A. Suppose that $(X, g)$ is a conformally compact Einstein 4-manifold with its conformal infinity of positive Yamabe constant and suppose the renormalized volume $V$ satisfies

$$
V>\frac{1}{3} \frac{4 \pi^{2}}{3} \chi(X)
$$

Then $X$ is homeomorphic to the 4 -ball $B^{4}$ up to a possible finite cover.

This result uses the vanishing theorems of Gursky [Gu2] to show that the doubling manifold $Y$ is a homology sphere, and the main result of [CGY1] to show that $Y$ admits a conformal metric of positive Ricci tensor, and hence has at most finite fundamental group. The homeomorphism classification theory of Donaldson and Freedman then implies that $Y$ is homeomorphic to the 4-sphere up to a finite cover. On the other hand, under a stronger hypothesis, we can conclude that $Y$ is diffeomorphic to the 4-sphere.

Theorem B. Suppose that $(X, g)$ is a conformally compact Einstein 4-manifold with conformal infinity of positive Yamabe constant. Then

$$
V>\frac{1}{2} \frac{4 \pi^{2}}{3} \chi(X)
$$

implies that $X$ is diffeomorphic to $B^{4}$ and $M$ is diffeomorphic to $S^{3}$.

These considerations demonstrate that the renormalized volume is an important conformal invariant of the conformally compact Einstein structure. The recent work of Fefferman and Graham [FG2] showed that it may be interpreted as a $Q$ curvature integral associated to a natural boundary operator. It would be of interest to understand this invariant. One may ask whether the positivity of the renormalized volume is an intrinsic property of the conformal structure of the boundary $M$ alone. This is related to the uniqueness question of the conformally compact Einstein structure. There is some partial answer to this question in the article of Anderson [A3] (cf. [Wa]).

The paper is organized as follows: In section 1 we introduce conformally compact Einstein manifolds and relevant properties. In section 2 we construct positive eigenfunctions and present a conformal compactification of a conformally compact Einstein manifolds. In section 3 we recall the results in [CGY1] [CGY2] [Gu1] [Gu2] and prove our main theorems. 


\section{Conformally Compact Einstein manifolds}

Let us start with the definition of a conformally compact Einstein manifold. Suppose $X^{n+1}$ is a compact oriented $(\mathrm{n}+1)$-manifold with boundary $\partial X=M^{n}$. A Riemannian metric $g$ in the interior of $X$ is said to be a $C^{m, \alpha}$ conformally compact if $\bar{g}=s^{2} g$ extends as a $C^{m, \alpha}$ metric on $\bar{X}$, where $s$ is a defining function of the boundary in the sense that: $s>0$ in $X, s=0$ and $d s \neq 0$ on $M$. Clearly defining functions are not unique. For a given defining function, the metric $\bar{g}$ restricted to $T M$ induces a metric $\hat{g}$ on $M$. $\hat{g}$ rescales upon changing the defining function $r$, therefore defines a conformal structure on $M$. We call $(M,[\hat{g}])$ the conformal infinity of the conformally compact manifold $(X, g)$. Therefore $\left(X^{n+1}, g\right)$ is a conformally compact Einstein manifold if it is conformally compact and $\operatorname{Ric}(g)=-n g$. A good reference for basic properties of conformally compact Einstein manifolds is the paper of Graham [Gr].

The boundary regularity of the conformally compact Einstein metric is an important issue. Thanks to M. Anderson [A2], in 4 dimension, we know that $C^{2, \alpha}$ would imply the full smoothness of the conformally compact Einstein metric. In other words, the conformally compact Einstein 4-manifolds we will be focus on in the last section are, always smooth when the conformal infinity is smooth, therefore, the local expansions of Einstein metrics near the infinity are always available. Sufficient boundary regularity is assumed in higher dimension to ensure the expansion though.

Solving a nonlinear first order PDE by the method of characteristics near the boundary, one has (cf. Lemma 2.1 and Lemma 2.2 in [Gr]):

Lemma 1.1. Given any conformally compact manifold $(X, g)$, a metric $\hat{g}$ in the conformal class $[\hat{g}]$ determines a unique defining function $s$ in a neighborhood of $M$ such that

$$
g=s^{-2}\left((d s)^{2}+g_{s}\right)
$$

where, when $n$ is odd,

$$
g_{s}=\hat{g}+g^{(2)} s^{2}+\text { even powers of } s+g^{(n-1)} s^{n-1}+g^{(n)} s^{n}+\cdots
$$

$g^{(i)}$ for $i<n$ are determined by local geometry of $(M, \hat{g})$ and $g^{(n)}$ is trace-free, when $n$ is even,

$$
g_{s}=\hat{g}+g^{(2)} s^{2}+\text { even powers of } s+h s^{n} \log s+g^{(n)} s^{n}+\cdots,
$$

$g^{(i)}$ for $i<n, h$ and the trace of $g^{(n)}$ are determined by local geometry of $(M, \hat{g})$, and $h$ is trace-free. 
For example, one can calculate that

$$
g^{(2)}=-\frac{1}{n-2}\left(\hat{R}_{i j}-\frac{\hat{R}}{2(n-1)} \hat{g}_{i j}\right) .
$$

As a realization of the so-called AdS/CFT correspondence, Henningson and Skenderis in $[\mathrm{HS}]$ and Graham in [Gr] defined and calculated the renormalized volume for a conformally compact Einstein manifold. Namely, they considered the expansions, when $n$ is odd,

$$
\operatorname{Vol}(\{s>\epsilon\})=c_{0} \epsilon^{-n}+c_{2} \epsilon^{-n+2}+\text { odd powers of } \epsilon+c_{n-1} \epsilon^{-1}+V+o(1)
$$

when $n$ is even,

$\operatorname{Vol}(\{s>\epsilon\})=c_{0} \epsilon^{-n}+c_{2} \epsilon^{-n+2}+$ even powers of $\epsilon+c_{n-2} \epsilon^{-2}+L \log \frac{1}{\epsilon}+V+o(1)$,

where

$$
c_{i}=\int_{M} v^{(i)} d v_{\hat{g}}
$$

and $v^{(i)}$ are local scalar invariants of $(M, \hat{g})$ of order $i$. More importantly he showed the renormalized volume $V$ for $n$ odd and $L$ for $n$ even are independent of the choice of defining functions. Incidentally when $n$ is even the quantity $L=\int_{M} v^{(n)} d v_{\hat{g}}$ is a conformal invariant of $M$, and when $n$ is odd, $V$ is only an invariant of the ambient structure $(X, g)$.

Particularly, when $n=3$, Anderson in [A] relates the renormalized volume to the Euler number of a conformally compact Einstein 4-manifold,

$$
8 \pi^{2} \chi(X)=\frac{1}{4} \int_{X}|\mathcal{W}|^{2} d v_{g}+6 V
$$

\section{Conformal COMpaCtifications}

In this section we present a conformal compactification of a general conformally compact Einstein manifold. As observed in [Q], when one considers the hemisphere compactification of the hyperbolic space, the conformal factor is a positive eigenfunction. Namely, recall

$$
\left(H^{n+1}, g_{H}\right)=\left(B^{n+1},\left(\frac{2}{1-|y|^{2}}\right)^{2}|d y|^{2}\right),
$$


and

$$
\left(S_{+}^{n+1}, g_{S}\right)=\left(B^{n+1},\left(\frac{2}{1+|y|^{2}}\right)^{2}|d y|^{2}\right),
$$

that is $g_{S}=t^{-2} g_{H}$, where we denote by

$$
t=\frac{1+|y|^{2}}{1-|y|^{2}}
$$

One finds after some calculation (cf. [Q]),

$$
\Delta_{g_{H}} t=(n+1) t .
$$

Given a general conformally compact Einstein manifold, using the theory of uniformly degenerate elliptic linear PDE developed in [M] [GL] [L], one can construct positive eigenfunctions. A good reference for our discussions here is a paper of Lee $[\mathrm{L}]$. The following lemma is a slight improvement of Lemma 5.2 in [L].

Lemma 2.1. Suppose that $\left(X^{n+1}, g\right)$ is a conformally compact Einstein manifold. Then there exists a positive function $u$ such that

$$
\Delta u=(n+1) u \text { in } X .
$$

In addition, when $n$ is odd,

$$
u=\frac{1}{s}+\frac{\hat{R}}{4 n(n-1)} s+w^{(4)} s^{3}+\text { odd powers of } s+w^{(n-1)} s^{n-2}+O\left(s^{n}\right)
$$

and when $n$ is even,

$$
u=\frac{1}{s}+\frac{\hat{R}}{4 n(n-1)} s+w^{(4)} s^{3}+\text { odd powers of } s+w^{(n)} s^{n-1}+O\left(s^{n}\right),
$$

where $w^{(i)}$ are all local invariants of Riemannian geometry of $(M, \hat{g})$ of order $i$.

Proof. To apply the theory of uniformly degenerate elliptic PDE (cf. [L], for example) we need to calculate

$$
\Delta \frac{1}{s}=\frac{s^{n+1}}{\sqrt{\operatorname{det} g_{s}}} \partial_{s}\left(s^{1-n} \sqrt{\operatorname{det} g_{s}} \partial_{s} \frac{1}{s}\right)=\frac{n+1}{s}-\frac{1}{2} \operatorname{Tr}_{\hat{g}} \partial_{s} g_{s} .
$$

Then from the expansion of $g_{s}$ obtained by Graham as stated in Lemma 1.1 in the previous section, when $n$ is odd,

$$
\Delta \frac{1}{s}=\frac{n+1}{s}+p^{(2)} s+\text { odd powers of } s+p^{(n-1)} s^{n-2}+O\left(s^{n}\right),
$$


and when $n$ is even,

$$
\Delta \frac{1}{s}=\frac{n+1}{s}+p^{(2)} s+\text { odd powers of } s+p^{(n)} s^{n-1}+O\left(s^{n}\right)
$$

where $p^{(k)}=-\frac{k}{2} \operatorname{Tr}_{\hat{g}} g^{(k)}$ are local invariants of Riemannian geometry of $(M, \hat{g})$ of order $k$, and

$$
p^{(2)}=\frac{\hat{R}}{2(n-1)},
$$

for example. Now let us calculate the key ingredient in the theory of uniformly degenerate elliptic PDE: the indicial equation. For that purpose we first calculate with separating variables for any smooth function $\phi$ defined on $(M, \hat{g})$,

$$
\Delta\left(\phi s^{k}\right)=\frac{\phi s^{n+1}}{\sqrt{\operatorname{det} g_{s}}} \partial_{s}\left(s^{1-n} \sqrt{\operatorname{det} g_{s}} \partial_{s} s^{k}\right)+\frac{s^{k+2}}{\sqrt{\operatorname{det} g_{s}}} \partial_{\alpha}\left(\sqrt{\operatorname{det} g_{s}} g_{s}^{\alpha \beta} \partial_{\beta} \phi\right)
$$

where

$$
\frac{\phi s^{n+1}}{\sqrt{\operatorname{det} g_{s}}} \partial_{s}\left(s^{1-n} \sqrt{\operatorname{det} g_{s}} \partial_{s} s^{k}\right)=k(k-n) \phi s^{k}+\frac{k}{2} \phi s^{k+1} \operatorname{Tr}_{\hat{g}} \partial_{s} g_{s}
$$

and

$$
\frac{s^{k+2}}{\sqrt{\operatorname{det} g_{s}}} \partial_{\alpha}\left(\sqrt{\operatorname{det} g_{s}} g_{s}^{\alpha \beta} \partial_{\beta} \phi\right)=s^{k+2} \tilde{\Delta} \phi+s^{k+2}(\cdots \text { even powers of } s \cdots)
$$

$\phi$ is independent of $s$, and $\tilde{\Delta}$ is the Laplacian of $(M, \hat{g})$. Therefore the indicial equation for us is

$$
(\Delta-(n+1))\left(\phi s^{k}\right)=(k(k-n)-(n+1)) \phi s^{k}+s^{k+2}(\cdots \text { even powers of } s \cdots)
$$

Then we may write, when $n$ is odd

$$
(\Delta-(n+1))\left(\frac{1}{s}+w^{(2)} s+\text { odd powers of } s+w^{(n-1)} s^{n-2}\right)=O\left(s^{n}\right)
$$

and when $n$ is even

$$
(\Delta-(n+1))\left(\frac{1}{s}+w^{(2)} s+\text { odd powers of } s+w^{(n)} s^{n-1}\right)=O\left(s^{n}\right) .
$$


Notice that $w^{(i)}$ are all local invariants of Riemannian geometry of $(M, \hat{g})$, for example for $i=2$ we have

$$
w^{(2)}=\frac{\hat{R}}{4 n(n-1)} .
$$

Then, the lemma follows from Proposition 3.3 in the paper of Lee [L]. Note that positivity of $u$ is a simple consequence of the maximum principle.

We remark that for the standard hyperbolic space we have

$$
u=t=\frac{1}{s}+\frac{1}{4} s .
$$

We can now calculate the scalar curvature for the metric $u^{-2} g$ on $X$ as in [Q]. We find, for $u$ obtained in Lemma 2.1,

$$
-\Delta u^{-\frac{n-1}{2}}-\frac{n^{2}-1}{4} u^{-\frac{n-1}{2}}=\frac{n^{2}-1}{4}\left(u^{2}-|d u|^{2}\right) u^{-\frac{n+3}{2}} .
$$

Thus the scalar curvature of $u^{-2} g$ is

$$
R\left[u^{-2} g\right]=n(n+1)\left(u^{2}-|d u|^{2}\right) .
$$

Notice that $u$ is determined by the choice of a defining function, therefore the choice of a metric of the conformal infinity by Lemma 1.1. So we may choose a Yamabe metric $\hat{g}$ for $(M,[\hat{g}])$ in the following theorem.

Theorem 2.2. Suppose that $\left(X^{n+1}, g\right)$ is a conformally compact Einstein manifold, and that $u$ is the eigenfunction obtained in Lemma 2.1 for a Yamabe metric $\hat{g}$ of the conformal infinity $(M,[\hat{g}])$. Then $\left(X^{n+1}, u^{-2} g\right)$ is a compact manifold with totally geodesic boundary $M$ and the scalar curvature is greater than or equal to $\frac{n+1}{n-1} \hat{R}$.

Proof. First by Lemma 2.1, one can estimate

$$
\begin{aligned}
u^{2} & -|d u|^{2}=\frac{1}{s^{2}}+\frac{\hat{R}}{2 n(n-1)}+O\left(s^{2}\right)-s^{2}\left(\partial_{s} u\right)^{2}-s^{2} g_{s}^{\alpha \beta} u_{\alpha} u_{\beta} \\
& =\frac{1}{s^{2}}+\frac{\hat{R}}{2 n(n-1)}-\frac{1}{s^{2}}+\frac{\hat{R}}{2 n(n-1)}+O\left(s^{2}\right) \\
& =\frac{\hat{R}}{n(n-1)}+O\left(s^{2}\right) .
\end{aligned}
$$

Thus in light of (2.6), the theorem follows from a maximum principle and the following Bochner formula,

$$
-\Delta\left(u^{2}-|d u|^{2}\right)=2|D d u-u g|^{2},
$$

which has also been used in $[\mathrm{L}]$. The smoothness assertion follows from the expansion (2.3). 


\section{Topology of CONFORMAlly COMPACT Einstein 4-MANifoldS}

In this section we apply the vanishing theorems of Gursky and the deformation theory of [CGY] of conformal metrics on the doubling 4-manifold $Y$ in order to draw conclusions about the topology of the conformally compact manifold $X$. Under increasingly stringent conditions on the renormalized volume $V$, we demonstrate the vanishing of $H^{1}(Y), H^{2}(Y)$. This is a reflection of the increasing constraint placed on the Weyl-Schouten tensor of the compactified structure.

According to the compactification obtained in Theorem 2.2 we have a compact 4-manifold $(X, \tilde{g})$ with totally geodesic boundary $M$. In addition the scalar curvatures satisfies $R[\tilde{g}] \geq 2 R[\hat{g}]$, where $\hat{g}$ is the Yamabe metric for the conformal infinity $(M,[\hat{g}])$. Recall the Chern-Gauss-Bonnet formula for 4-manifolds with totally geodesic boundary

$$
8 \pi^{2} \chi(X)=\int_{X}\left(\frac{1}{4}|\tilde{\mathcal{W}}|^{2}+\sigma_{2}\left(A_{\tilde{g}}\right)\right) d v_{\tilde{g}}
$$

where

$$
\sigma_{2}(A)=\frac{1}{24} R^{2}-\frac{1}{2}|E|^{2}
$$

and $E$ is the traceless Ricci curvature. It follows from the conformal invariance

$$
|\tilde{\mathcal{W}}|^{2} d v_{\tilde{g}}=|\mathcal{W}|^{2} d v_{g}
$$

and Anderson's formula (1.8) that

$$
\int_{X} \sigma_{2}\left(A_{\tilde{g}}\right) d v_{\tilde{g}}=6 V
$$

Consider the doubling manifold $(Y, \bar{g})$ of $(X, \tilde{g})$. The metric $\bar{g}$ on $Y$ belongs to $C^{2,1}$, i.e. its second derivative is of Lipschitz class, therefore the curvature tensor is of Lipschitz class. Consequently we have

Proposition 3.1. If the conformal infinity has positive Yamabe invariant, then the renormalized volume satisfies

$$
V \leq \frac{4}{3} \pi^{2}
$$

Equality in (3.4) holds if and only if $(X, g)$ is the hyperbolic space, therefore $(M,[\hat{g}])$ is the round sphere. 
Proof. According to Gursky ([Gu1]) if a 4-manifold $(Y, \bar{g})$ is of positive Yamabe constant, then

$$
\int_{Y} \sigma_{2}\left(A_{\bar{g}}\right) d v_{\bar{g}} \leq 16 \pi^{2}
$$

and equality holds if and only if $(Y, \bar{g})$ is the round 4-sphere. In the situation at hand, the doubling space $Y$ has a metric of positive scalar curvature according to Theorem 2.2, therefore,

$$
V \leq \frac{1}{6} \int_{X} \sigma_{2}(\tilde{g}) d v_{\tilde{g}} \leq \frac{1}{6} 8 \pi^{2}=\frac{4}{3} \pi^{2}
$$

and equality holds if and only if

$$
\int_{X}|\mathcal{W}|^{2} d v_{g}=0
$$

In this case the simply connected hyperbolic manifold is the hyperbolic space, and the conformal infinity is the round sphere. The proof is complete.

Let us assume that $(X, g)$ is a conformally compact Einstein 4-manifold whose conformal infinity $(M,[\hat{g}])$ has positive Yamabe invariant. According to the spectral result of Lee in $[\mathrm{L}]$ and a vanishing result of Wang [Wa](cf. [CG] [WY]), there are no harmonic one forms in $L^{2}$. Therefore it follows from the isomorphism established by Mazzeo [M], the space of $L^{2}$ harmonic one forms is isomorphic to the relative cohomology $H^{1}(X, M)$, hence the latter has to vanish.

We have the long exact sequence in cohomology for the pair $(X, M)$ :

$$
\ldots H^{k}(X, M) \stackrel{j^{*}}{\longrightarrow} H^{k}(X) \stackrel{i^{*}}{\longrightarrow} H^{k}(M) \stackrel{\delta^{*}}{\longrightarrow} H^{k+1}(X, M) \ldots
$$

and the Mayer-Vietoris sequence for the double $Y=X \cup X^{\prime}$ where $X^{\prime}$ is the second copy of $X$ :

$$
\ldots H^{k-1}(M) \stackrel{\gamma}{\longrightarrow} H^{k}(Y) \stackrel{\psi^{*}}{\longrightarrow} H^{k}(X) \oplus H^{k}\left(X^{\prime}\right) \stackrel{\phi^{*}}{\longrightarrow} H^{k}(M) \ldots
$$

Since $H^{1}(X, M)=0$, it follows from the long exact sequence that $i^{*} H^{1}(X)=$ $H^{1}(X)$. The Mayer-Vietoris sequence then implies that $H^{1}(Y)=H^{1}(X)$.

We wish to impose a second positivity assumption on the conformally compact Einstein structure in addition to the positivity of Yamabe invariant of the boundary: the renormalized volume $V$ should be positive. We remark that this implies in view of the formula $(0.1)$ that $\chi(X)>0$. In fact we have a stronger consequence according to the first vanishing theorem of Gursky [Gu2]: 
Theorem 3.2. [Gu2] If $(N, g)$ is a closed 4-manifold with positive Yamabe invariant, and

$$
\int_{N} \sigma_{2}\left(A_{\bar{g}}\right) d v_{\bar{g}}>0
$$

then $H^{1}(N)=0$.

In order to apply the vanishing theorem, we need to assure ourselves that the doubling metric is smooth. Since the metric on $X$ is of the class $C^{2,1}$ and the boundary is totally geodesic, we may (if necessary) smooth out the metric by a small perturbation without changing the two positivity assumptions: positive Yamabe invariant and $\int_{Y} \sigma_{2}\left(A_{\bar{g}}\right) d v_{\bar{g}}>0$. We conclude thus $H^{1}(X)=H^{1}(Y)=0$.

Next we formulate a stronger positivity condition in order to control the second homology. Recall for a closed 4-manifold $(Y, \bar{g})$, we have the Chern-Gauss-Bonnet formula $(0,2)$. On the other hand we also have the Hirzebruch signature formula

$$
12 \pi^{2} \tau(Y)=\int_{Y} \frac{1}{4}\left(\left|\overline{\mathcal{W}}^{+}\right|^{2}-\left|\overline{\mathcal{W}}^{-}\right|^{2}\right) d v_{\bar{g}}
$$

Combining the two formulae we have

$$
4 \pi^{2}(2 \chi(Y)+3 \tau(Y))=\frac{1}{4} \int_{Y}\left|\overline{\mathcal{W}}^{+}\right|^{2} d v_{\bar{g}}+\int_{Y} \sigma_{2}\left(A_{\bar{g}}\right) d v_{\bar{g}}
$$

and

$$
4 \pi^{2}(2 \chi(Y)-3 \tau(Y))=\frac{1}{4} \int_{Y}\left|\overline{\mathcal{W}}^{-}\right|^{2} d v_{\bar{g}}+\int_{Y} \sigma_{2}\left(A_{\bar{g}}\right) d v_{\bar{g}}
$$

Theorem 3.3. [Gu2] Suppose that $(Y, \bar{g})$ is a closed oriented 4-manifold with positive Yamabe constant. Then

$$
\frac{1}{4} \int_{Y}\left|\overline{\mathcal{W}}^{+}\right|^{2} d v_{\bar{g}}<\int_{Y} \sigma_{2}\left(A_{\bar{g}}\right) d v_{\bar{g}}
$$

implies that the self-dual part of the second homology vanishes; similarly,

$$
\frac{1}{4} \int_{Y}\left|\overline{\mathcal{W}}^{-}\right|^{2} d v_{\bar{g}}<\int_{Y} \sigma_{2}\left(A_{\bar{g}}\right) d v_{\bar{g}}
$$

implies that the anti-self-dual part of the second homology vanishes. 
Proposition 3.4. Assume that $(X, g)$ is a conformally compact Einstein 4 manifold whose conformal infinity has positive Yamabe invariant and that

$$
V>\frac{1}{3} \frac{4 \pi^{2}}{3} \chi(X)
$$

then $V>0$ and $H^{2}(X, \mathbb{R})=0$.

Proof. According to (1.8),

$$
\frac{1}{4} \int_{X}|\tilde{\mathcal{W}}|^{2} d v_{\tilde{g}}=8 \pi^{2} \chi(X)-6 V<12 V
$$

Therefore $V>0$. If we translate the assumption (0.3) into assumption on the doubling manifold $(Y, \bar{g})$, we have

$$
\int_{Y} \sigma_{2}\left(A_{\bar{g}}\right) d v_{\bar{g}}>\frac{8}{3} \pi^{2} \chi(Y)
$$

here we note that $\chi(Y)=2 \chi(X)$. It follows from (0.2) that (3.15) is equivalent to

$$
\frac{1}{4} \int_{Y}|\overline{\mathcal{W}}|^{2} d v_{\bar{g}}<2 \int_{Y} \sigma_{2}\left(A_{\bar{g}}\right) d v_{\bar{g}}
$$

At this point, we remark once more that we may smooth the doubling metric if necessary while preserve the condition (3.16).

We can now assume without loss of generality that

$$
\frac{1}{4} \int_{Y}\left|\overline{\mathcal{W}}^{+}\right|^{2} d v_{\bar{g}}<\int_{Y} \sigma_{2}\left(A_{\bar{g}}\right) d v_{\bar{g}}
$$

and apply the vanishing theorem Theorem 3.2 of Gursky, and conclude that $H_{+}^{2}(Y)$ $=0$. From (3.11) we also have

$$
4 \pi^{2}(2 \chi(Y)+3 \tau(Y)) \geq \int_{Y} \sigma_{2}\left(A_{\bar{g}}\right) d v_{\bar{g}}>\frac{8}{3} \pi^{2} \chi(Y) .
$$

A short computation with the two long exact sequences (3.7) and (3.8) and using the fact $H^{1}(X)=H^{1}(Y)=0$ will yield that $H^{2}(Y)=H^{2}(X) \oplus H^{2}\left(X^{\prime}\right)$ and hence $\operatorname{dim} H^{2}(Y)$ is even. Hence we we may assume $\operatorname{dim} H_{-}^{2}(Y)=2 k$. Then (3.18) yields

$$
2(2+2 k)+3(-2 k)>\frac{2}{3}(2+2 k)
$$

which is impossible for any positive integer $k$. Therefore $k=0$ and $H^{2}(X, \mathbb{R})=$ $H^{2}(Y, \mathbb{R})=0$ and we have finished the proof of the proposition.

So far we have applied the vanishing theorems of Gursky to conformally compact Einstein manifolds. Next we develop the implication the recent work of ChangGursky-Yang [CGY1] [CGY2] on the doubling manifold $Y$. 
Theorem 3.5. [CGY1] Suppose that $(Y, \bar{g})$ is an oriented closed 4-manifold with positive Yamabe constant and that

$$
\int_{Y} \sigma_{2}\left(A_{\bar{g}}\right) d v_{\bar{g}}>0 .
$$

Then there exists a conformal metric $g^{\prime}=e^{2 w} \bar{g}$ with positive Ricci tensor.

\section{Proof of Theorem A}

As a consequence of Theorem 3.5, we can conclude that the doubling manifold $Y$ has a finite fundamental group. If we consider its simply connected covering $\tilde{Y}$, the homeomorphic classification theory of Donaldson and Freedman for simply connected 4-manifolds implies that the manifold $\tilde{Y}$ is determined by its intersection form. It follows from Proposition 3.4 that $H^{2}(Y, \mathbb{R})=0$ under the assumption $(0.3)$ of Theorem A. The vanishing of $H^{2}(Y, \mathbb{R})$ in turn implies that $\tilde{Y}$ is homeomorphic to $S^{4}$. We have thus established Theorem A.

We will now discuss the condition (0.4) in Theorem B which is stronger than the condition (0.3) in the statement of Theorem A. To see the implication of (0.4), we first recall a main result in [CGY2].

Theorem 3.6. [CGY2] Suppose that $(Y, \bar{g})$ is an oriented closed 4-manifold with positive Yamabe constant and that

$$
\frac{1}{4} \int_{Y}|\overline{\mathcal{W}}|^{2} d v_{\bar{g}}<\int_{Y} \sigma_{2}\left(A_{\bar{g}}\right) d v_{\bar{g}} .
$$

Then $Y$ is diffeomorphic to $S^{4}$.

\section{Proof of Theorem B}

Again we will use the doubling manifold $(Y, \bar{g})$. We know by our assumption (0.4) that $(Y, \bar{g})$ is a $C^{2,1}$ closed doubling 4-manifold with positive scalar curvature and

$$
\frac{1}{4} \int_{Y}|\overline{\mathcal{W}}|^{2} d v_{\bar{g}}<\int_{Y} \sigma_{2}\left(A_{\bar{g}}\right) d v_{\bar{g}} .
$$

We observe that the smoothness condition $\bar{g} \in C^{2,1}$ means that we can find a nearby smooth metric $g_{0}$ of $\bar{g}$ such that the above mentioned three properties all preserved. Now we are ready to apply the argument in [CGY1] [CGY2] to deform $g_{0}$ into the metric of the standard 4-sphere with the doubling property preserved all the 
way. For this purpose we want to sketch the ideas given in [CGY1] [CGY2] in the following.

First, one considers the following functional $F: W^{2,2}(Y) \longrightarrow \mathbb{R}$ :

$$
F[\omega]=\tilde{\gamma}_{1} \tilde{I}[\omega]+\gamma_{1} I[\omega]+\gamma_{2} I I[\omega]+\gamma_{3} I I I[\omega]
$$

For our situation we need to consider an even nowhere-vanishing symmetric $(0,2)$ tensor $\eta$ which may be taken as the metric $g_{0}$ for example. For particular choice of the coefficients

$$
\begin{aligned}
\tilde{\gamma}_{1} & =-\frac{\int_{Y} \sigma_{2}\left(A_{0}\right) d v_{0}-\int_{Y}\left|\mathcal{W}_{0}\right|^{2} d v_{0}}{2 \int_{Y}\left|\eta_{0}\right|^{2} d v_{0}}<0, \\
\gamma_{1} & =-\frac{1}{8} \\
\gamma_{2} & =1 \\
\gamma_{3} & =\frac{3 \delta-2}{24}>0
\end{aligned}
$$

following the work of [CY], it is proved in [CGY1] [CGY2] that there exists smooth function $\omega$ which achieves the infimum of $F[\omega]$ over $W^{2,2}(Y)$ and satisfies

$$
\sigma_{2}(A)-|\mathcal{W}|^{2}=\frac{\delta}{4} \Delta R-\tilde{\gamma}_{1}|\eta|^{2}
$$

for all $\delta>\frac{2}{3}$, here we need to emphasize that terms in (3.23) are taken with respect to the metric $e^{2 \omega} g_{0}$. Second, by a priori estimate and non-degeneracy of the linearization of (3.23) (cf. [CGY1] [CGY2]) one may conclude that there exists metric $e^{2 \omega_{\delta}} g_{0}$ which satisfies (3.23) for all $\delta>0$. Third, take a metric $e^{2 \omega_{\delta}} g_{0}$ obtained by the previous steps for sufficient small $\delta$ and deform it by Yamabe flow in the conformal class. It was proved in [CGY1] [CGY2] that, after some time, the metrics along the Yamabe flow satisfy

$$
\sigma_{2}(A)-\frac{1}{4}|\mathcal{W}|^{2}>0
$$

Finally, one applies a weak pinching result of Margerin [Ma]. Namely, with (3.24), one can deform again the metric by Ricci flow into the standard metric for the round 4-sphere (cf. Section 2 in [CGY2]).

Finally we need to make sure that in each of the above steps the doubling symmetry is preserved. For this purpose we simply make sure that the solution in each step is unique, at least locally. For the first step, one may use the uniqueness of the 
extremal of $F$, because of $\kappa=0$ (cf. Theorem 2.1 in [CY]), to conclude that the extremal of $F$ has to be an even function on the doubling manifold $\left(Y, g_{0}\right)$. In second step, we have a local uniqueness as the linearized equation is non-degenerate, which also assures the solution $\omega_{\delta}$ obtained in the argument in [CGY1] [CGY2] is even for all $\delta>0$. The last two steps are clear since the uniqueness of the two geometric flows guarantees that all metrics along the flows respect the doubling symmetry if the initial metrics are. Thus the proof is completed.

\section{References:}

[A1] M. Anderson, $L^{2}$ curvature and volume renormalization of the AHE metrics on 4-manifolds, Math. Res. Lett., 8 (2001) 171-188.

[A2] M. Anderson, Boundary regularity, uniqueness and non-uniqueness for AH Einstein metrics on 4-manifolds, preprint.

[A3] M. Anderson, Einstein metrics with prescribed conformal infinity on 4manifolds, preprint.

[CG] M. Cai and G.J. Galloway, Boundary of zero scalar curvature in the AdS/CFT correspondence, Preprint hep-th/0003046.

[CY] S.-Y. A. Chang and P. Yang, Extremal metrics of zeta functional determinants on 4-manifolds, Ann. of Math. 142(1995) 171-212.

[CGY1] S.-Y. A. Chang, M. Gursky and P. Yang, An equation of Monge-Ampere type in conformal geometry and 4-manifolds of positive Ricci curvature, Annals of Math. 155 (2002), 709-787.

[CGY2] S.-Y. A. Chang, M. Gursky and P. Yang, A conformally invariant Sphere theorem in four dimension, Preprint 2002.

[FG1] C. Fefferman, and C.R. Graham, Conformal invariants, in The mathematical heritage of Elie Cartan, Asterisque, 1985, 95-116.

[FG1] C. Fefferman, and C.R. Graham, Q-curvature and Poincare metrics, Math. Res. Lett., 9 (2002), no. 2-3, 139-151.

[Gr] C. R. Graham, Volume and Area renormalizations for conformally compact Einstein metrics. The Proceedings of the 19th Winter School "Geometry and Physics" (Srní, 1999). Rend. Circ. Mat. Palermo (2) Suppl. No. 63 (2000), 31-42.

[GL] C.R. Graham and J. Lee, Einstein metrics with prescribed conformal infinity 
on the ball. Adv. Math. 87 (1991), no. 2, 186-225.

[GKP] S.S.Gubser, I.R.Klebanov and A.M.Polyakov, Gauge theory correlators from non-critical string theory, Phys Lett. B 428 (1998) 105-114, hep-th/9802109

[Gu1] M. Gursky, The principal eigenvalue of a conformally invariant differential operator, with application to semilinear elliptic PDE, Comm. Math. Phys. 207 (1999), 131-143.

[Gu2] M. Gursky, The Weyl functional, de Rham cohomology, and Kähler-Einstein metrics. Ann. of Math. (2) 148 (1998), no. 1, 315-337.

[HS] M. Henningson and K. Skenderis, The holographic Weyl anomaly, J. High. Energy Phys. 07 (1998) 023 hep-th/9806087; Holography and the Weyl anomaly hep-th/9812032

[L] J. Lee, The spectrum of an asymptotically hyperbolic Einstein manifold. Comm. Anal. Geom. 3 (1995), no. 1-2, 253-271.

[Mc] J. Maldacena, The large N limit of superconformal field theories and supergravity, Adv. Theo. Math. Phy. 2 (1998) 231-252, hep-th/9711200

[Ma] C. Margerin, A sharp characterization of the smooth 4-sphere in curvature forms; CAG, 6 (1998), no.1, 21-65.

[M] R. Mazzeo. The Hodge cohomology of a conformally compact metric, J. Diff. Geom. 28(1988) 309-339.

[Q] J. Qing, On the rigidity for conformally compact Einstein manifolds, Preprint 2002.

[Wa] X. Wang, On conformally compact Einstein manifolds, Math. Res. Lett. 8(2001), no. 5-6, 671-688.

[W] E. Witten, Anti-de Sitter space and holography, Adv. Theo. Math. Phy. 2 (1998) 253-290, hep-th/9802150

[WY] E. Witten and S.T. Yau, Connectedness of the boundary in ADS/CFT correspondence, Preprint het-th/9910245. 